\title{
A Novel Particle Jump Particle Swarm Optimization Method for PV MPPT Control under Partial Shading Conditions
}

\author{
Bingcheng $\mathrm{Ji}^{* \text { a) }}$ \\ Takehiro Imura*** \\ Non-member, \\ Katsuhiro Hata** \\ Member \\ Sayuri Honda*** \\ Member, \\ Yoichi Hori* \\ Fellow \\ Non-member, \\ Shuhei Shimada $a^{* * *}$ \\ Non-member \\ Osamu Kawasaki ${ }^{* * * *}$ Non-member
}

(Manuscript received Nov. 11, 2019, revised Dec. 19, 2019)

\begin{abstract}
With the sharp increase in the global energy demands, photovoltaics (PV) have been developed significantly in recent years. However, when the PV work under partial shading conditions, the global maximum power point tracking control should be executed. In this paper, a novel particle swarm optimization (PSO) algorithm with the particle jump improvement is proposed to track the global maximum $(G M)$ of PV output power under partial shading conditions. In the proposed method, each particle is allocated within respective intervals at initial iteration suh that the particle only explores the corresponding interval to determine the potential $G M$. When the corresponding interval has been traversed without determining the $G M$, the interval will be discarded and the particle will jump to the interval where the current tracked $G M$ is present. Therefore, each interval of the converter duty range will be traversed by only one particle and the total algorithm tracking time will be reduced. The proposed algorithm is verified with a simulation and an experiment. Based on the experimental results, the tracking times of the proposed method are $0.8 \mathrm{~s}, 0.8 \mathrm{~s}$, and $1.2 \mathrm{~s}$ when the PV output power possesses 2, 3, and 4 peaks under partial shading conditions, respectively.
\end{abstract}

Keywords: particle swarm optimization, global maximum power point tracking, particle jump, photovoltaic, simulated annealing

\section{Introduction}

In recent years, all of the population, technology and economy have achieved great advancements all over the world, and the energy consumption has also approached a sharp increase $^{(1)-(3)}$. Considering this issue, the research on renewable energy resource has attracted the worldwide attention and some significant developments have been established in various areas ${ }^{(4)}$. Among all the renewable energy resources which are focused, due to the high accessibility and inexhaustibility of solar energy, the PV has been regarded as one of the most promising candidates for the future energy sources ${ }^{(5)}$. The PV has been employed in various areas, such as the power grid, electric vehicle as well as space exploration, and it has brought about a great deal of improvements to the corresponding applications ${ }^{(6)}$.

a) Correspondence to: Bingcheng Ji. E-mail: ki.heisei17@ae.k. u-tokyo.ac.jp

* Department of Advanced Energy, Graduate School of Frontier Sciences, University of Tokyo

5-1-5, Kashiwa, Chiba 277-8561, Japan

${ }^{* *}$ Department of Informatics and Electronics, Institute of Industrial Science, University of Tokyo

4-6-1, Komaba, Tokyo 153-8505, Japan

*** Department of Electrical Engineering, Tokyo University of Science

2641, Yamazaki, Chiba 278-8510, Japan

${ }^{* * * *}$ Reseach Unit I, R\&D Directorate, Japan Aerospace Exploration Agency

2-1-1, Sengen, Tsukuba 305-8505, Japan
In order to make the most of the solar irradiance, the PV maximum power point tracking (MPPT) should be conducted under working conditions ${ }^{(7)}$. Due to the PV property, when the solar irradiance on the PV panel is uniform, the PV output power with respect to the voltage only possesses one peak. On the other hand, when the solar irradiance is not uniform, namely the PV panel is under partial shading conditions, there will be multiple peaks at the power-voltage curve ${ }^{(8)}$. Therefore, considering the solar irradiance, the PV MPPT algorithms also can be divided into two categories, namely the local maximum tracking algorithm and global maximum tracking algorithm.

When the solar irradiance is uniform, because the PV output power only possesses one peak, the local maximum tracking algorithms, such as the Perturb\&Observe (P\&O), Incremental Conductance (InCon) and Constant Voltage (CV) methods, can be employed ${ }^{(9)}$. For these methods, the computation cost and hardware requirement are low, and they are simple to be conducted. However, under working conditions, because of the shadows of clouds, buildings or trees, the solar irradiance on the PV panel is not possible to be kept uniform all the time. Therefore, in this case, the global MPPT algorithms should be employed to search the $G M$ among the multiple local maximums $(L M s)^{(10)}$. So far, there have been various $G M$ tracking algorithms developed in the PV area, such as the Particle Swarm Optimization (PSO) ${ }^{(11)-(13)}$, Flower Pollination Algorithm (FPA) ${ }^{(14)(15)}$, Differential Evolution (DE) ${ }^{(16)(17)}$, Genetic Algorithm (GA) ${ }^{(18)(19)}$, Firefly Algorithm (FA) ${ }^{(20)(21)}$ and Grey Wolf Optimizer $(\mathrm{GWO})^{(22)(23)}$ 


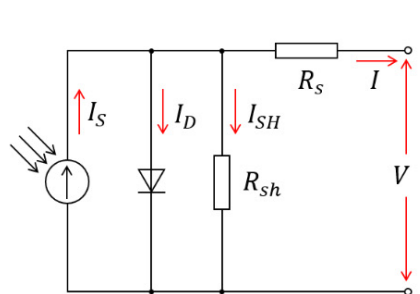

(a)

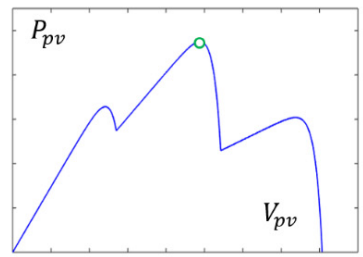

(e)

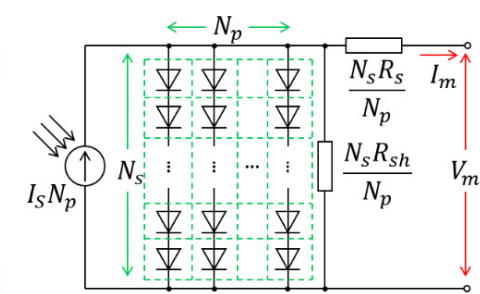

(b)

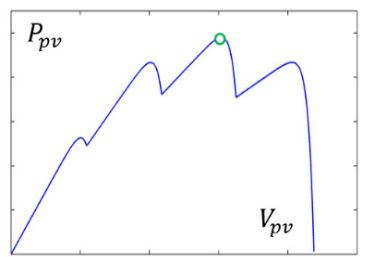

(f)

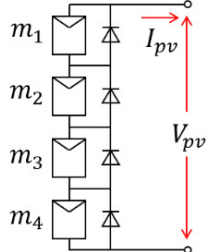

(c)

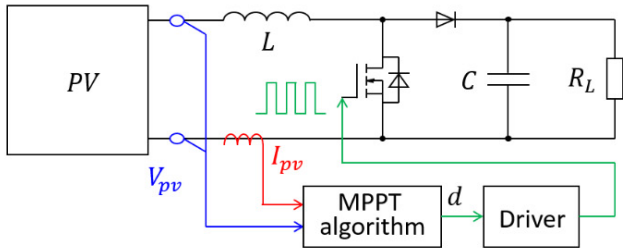

(g)

Fig. 1. (a) PV cell one-diode model. (b) PV module structure. (c) Employed PV panel structure in this paper. (d) 2-peak PV output property. (e) 3-peak PV output property. (f) 4-peak PV output property. (g) Employed MPPT control converter topology

algorithms.

A novel enhanced leader PSO algorithm has been proposed $^{(24)}$, and due to the successive particle mutations the proposed method has exhibited fast convergence and better dynamic performance of PV MPPT control under partial shading conditions. A FPA algorithm is proposed and exhibits quick convergence capability in strong shaded conditions due to the randomness crested in the local and global pollinations in the MPPT process ${ }^{(25)}$. Furthermore, the PV output power also achieves high stability due to the iteration optimization. An improved DE algorithm with the whale optimization is proposed $^{(26)}$, and it can jump out of the local MPP stagnation and reduce the algorithm iterations. Compared with the conventional DE methods, the proposed algorithm can reduce the $G M$ searching time and improve the convergence performance. An artificial neural network (ANN) optimized GA algorithm is proposed ${ }^{(27)}$, and GA is employed to extract the MPP from PV output to conduct the ANN offline training. Under working conditions, the proposed algorithm can deal with the fast changing solar irradiance conditions because the ANN can produce the reference voltage corresponding to the MPP for any environmental change. A FA method is proposed to conduct the fast MPPT control in partially shaded PV arrays ${ }^{(28)}$. The tracking process consists of positioning the fireflies in the possible solution space in the initial stage and based on the PV power moving the fireflies to the promising regions in the following. The experiment results demonstrate the proposed method can jump out of the current MPPT point and reorient toward new $G M$ under rapidly changing partial shading conditions. A hybrid MPPT algorithm of GWO and $\mathrm{P} \& \mathrm{O}$ is proposed to cope with the rapid variations of solar irradiance and partial shading conditions ${ }^{(29)}$. In the proposed method, GWO is employed to determine the rough position range of $G M$ and $\mathrm{P} \& \mathrm{O}$ is used to achieve the fast convergence to the global peak.

However, all the introduced papers face the same issue: the same particle range may be traversed by some particles repeatedly and this will result in longer tracking time. Based on the above analysis, in this paper, a novel particle jump PSO algorithm is proposed for PV to track the $G M$ under partial shading conditions. The proposed algorithm consists of two parts: particle jump and PSO stages. At the first stage, the particle jump process can achieve the rough $G M$ positioning and at the second stage, PSO can conduct the fast convergence to the global peak. The paper is organized as follows: section 2 analyzes the PV output property; section 3 introduces the PSO algorithm; section 4 proposes the particle jump method; section 5 expresses the particle jump dectection method; section 6 and 7 conduct the simulation and experiment; section 8 presents the conlcusion.

\section{PV Model and Output Property Analysis}

In order to enhance the PV output power, there are a great deal of PV cells connected in series and parallel in a PV panel. In this paper, the one-diode PV cell model in Fig. 1(a) is employed to analyze the PV output current ${ }^{(30)}$. Based on the model, the output current of a PV cell can be obtained as follows.

$$
I=I_{S}-I_{0} \cdot\left[\exp \left(\frac{V+R_{s} \cdot I}{n \cdot V_{t}}\right)-1\right]-\frac{V+R_{s} \cdot I}{R_{s h}} \cdots \cdots
$$

In the equation, $V$ and $I$ are the PV cell output voltage and current, respectively; $I_{S}$ is the light generated current; $V_{t}$ it the junction thermal voltage; $I_{0}$ is the leakage or reverse saturation current; $n$ is the diode quality factor; $R_{S}$ is the series resistance; $R_{s h}$ is the shunt resistance. Furthermore, the light generated current $I_{S}$ can be obtained as follows.

$$
I_{S}=\frac{S}{S_{r e f}}\left[I_{S, r e f}+\alpha_{s c}\left(T-T_{r e f}\right)\right] \ldots \ldots \ldots \ldots \ldots \ldots
$$

In the equation, ref stands for the reference conditions; $S$ and $S_{\text {ref }}$ are the solar irradiance intensity and reference $\left(1000 \mathrm{~W} / \mathrm{m}^{2}\right)$, respectively; $T$ and $T_{r e f}$ are the panel temperature and reference $\left(25^{\circ} \mathrm{C}\right)$, respectively; $I_{S, \text { ref }}$ is the PV cell output current under the reference standards of $S_{\text {ref }}$ and $T_{\text {ref }}$; $\alpha_{s c}$ is the absolute temperature coefficient of short circuit current.

In addition, the diode reverse saturation current $I_{0}$ can be obtained as follows. 
Table 1. PV property under partial shading conditions

\begin{tabular}{cccc}
\hline Case & $V_{\text {opt }} / \mathrm{V}$ & $I_{\text {opt }} / \mathrm{A}$ & $P_{\max } / \mathrm{W}$ \\
\hline 1 & 27.61 & 1.77 & 48.98 \\
\hline 2 & 24.40 & 1.95 & 47.53 \\
\hline 3 & 30.37 & 1.61 & 49.02 \\
\hline
\end{tabular}

$$
I_{0}=I_{0, r e f}\left[\frac{T}{T_{r e f}}\right]^{3} \exp \left[\frac{E_{g, r e f}}{k_{b} T_{r e f}}-\frac{E_{g}}{k_{b} T}\right] \ldots \ldots \ldots \ldots
$$

In the equation, $I_{0, \text { ref }}$ is the diode reverse saturation current under reference condition; $k_{b}$ is the Boltzmann constant of $k_{b}=1.3806503 e^{-23} \mathrm{~J} / \mathrm{K} ; E_{g}$ and $E_{g, r e f}$ are the bandgap energy of the silicon and reference, respectively.

Because the output of one PV cell is really weak, in applications the PV cells are always connected in series and parallel to yeild the PV module model in Fig. 1(b). In the PV module model, $V_{m}$ and $I_{m}$ are the PV module output voltage and current, respectively. Then the PV modules are further connected in network to generate the PV panel in Fig. 1(c). Based on the PV panel model, $V_{p v}$ and $I_{p v}$ are the PV panel output voltage and current, respectively. Because the PV modules are connected in series, in this paper, it can be concluded that the PV panel voltage, current and power are $V_{p v}=4 V_{m}, I_{p v}=I_{m}$ and $P_{p v}=V_{p v} I_{p v}$, respectively. Based on the above model analysis, when the solar irradiance on the PV panel is not uniform under partial shading conditions, the PV output power will possess multiple peaks. In order to make the most use of solar energy, the global maximum of PV output power should be tracked. In this paper, the PV output properties in Fig. 1(d), (e), (f) and Table 1 are employed to verify the proposed global MPPT tracking algorithm.

Based on the PV property, when the system equivalent resistance looking from the PV is equal to be the optimal value the PV maximum power can be tracked. In this paper, in order to transform the system equivalent resistance, the converter in Fig. $1(\mathrm{~g})$ is employed. The converter switching frequency is $20 \mathrm{kHz}$, and the inductor $L$, capacitor $C$ and load $R_{L}$ are $1 \mathrm{mH}, 1000 \mu \mathrm{F}$ and $5 \Omega$, respectively.

\section{Particle Swarm Optimization Algorithm}

The PSO algorithm is a method which employs an amount of particles simulating the social behaviors of animal groups to explore the global optimal position. Under working conditions, the particle movement in PSO algorithm is achieved by the particle velocity and position updates. The corresponding velocity and position update equations are shown as follows.

$$
\begin{aligned}
& v_{i}^{(k+1)}=w v_{i}^{(k)}+c_{1} r_{1}\left(P_{M i}-x_{i}^{(k)}\right)+c_{2} r_{2}\left(G_{M}-x_{i}^{(k)}\right) \cdots \\
& x_{i}^{(k+1)}=x_{i}^{(k)}+v_{i}^{(k+1)} \ldots \ldots \ldots \ldots \ldots \ldots \ldots \ldots \ldots \ldots \ldots \ldots \ldots \ldots
\end{aligned}
$$

In the velocity update of Eq. (4): $w$ is the inertia weight which determines how much the particle current state is influenced by the last state; $P_{M i}$ is the personal maximum $(P M)$ position of the $i^{\text {th }}$ particle; $G_{M}$ is the $G M$ position; $c_{1}$ and $c_{2}$ are the scaling factors which determine how much the particle tendentiousness is between $P_{M i}$ and $G_{M}$. Higher $c_{1}$ means the particle movement is more influenced by the $P M$ and then the real $G M$ position is more possible to be tracked, but the tracking time will be prolonged. Higher $c_{2}$ means the particle movement is more influenced by the current $G M$ position.
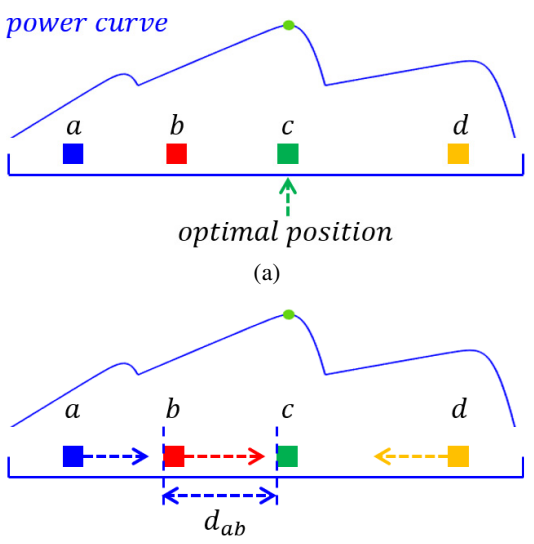

(b)

Fig. 2. (a) Particle initialization in the converter duty range. (b) Particle movement in MPPT process

The algorithm convergence time will be reduced but the probability of tracking the real $G M$ position also decreases; $r_{1}$ and $r_{2}$ are the random values following the uniform distribution $U(0,1)$. In the position update of Eq. (5), with the current velocity update, the particle position can be updated.

In each iteration, all the particles will be employed as the converter duty to obtain the corresponding fitness, namely the PV power. In the algorithm conducting process, the $P M$ positions and $G M$ position are updated based on all the particle positions and fitness. Furthermore, with the guidance of the personal and $G M$ positions, all the particles will gradually move toward the $G M$ position. After the PSO algorithm iterates enough times, the $G M$ can be effectively tracked.

\section{Particle Jump Method Proposal}

Because of the capability of tracking the $G M$, the PSO algorithm has been employed into various areas. However, as shown in Eq. (5), the particle position in the PSO algorithm is updated step by step under working conditions. On one hand, because all the particle positions are updated gradually with velocity increments, the $G M$ position is highly possible to be tracked with appropriate parameters of $w, c_{1}$ and $c_{2}$. On the other hand, in general, the velocity increment is always limited within an appropriate range to reduce the algorithm fluctuation, and then the PSO algorithm will take too much time to achieve the convergence of all the employed particles.

4.1 Invalid Interval Traversal Analysis in PSO Algorithm Due to the PV property, under partial shading conditions, the PV output power with respect to voltage is a twodimension curve, and the particle position is the converter duty varying in the range of $(0,1)$, as shown in Fig. 2(a). There are 4 particles of $a, b, c$ and $d$ are employed and the $G M$ is at the position of particle $c$.

In the MPPT process, both particle $a$ and $b$ move to the right side to the global maximum power point and suppose the distance is traversed by particle $a$ and $b$ in succession as shown in Fig. 2(b). However, there are two points should be mentioned: (1) because the initial positions and velocities of particle $a$ and $b$ are different, the possibility that particle $a$ and $b$ traverse the duty range $d_{a b}$ with the same position and velocity at each iteration is low enough; (2) the distance $d_{a b}$ is traversed twice by the particle $a$ and $b$, and the former 


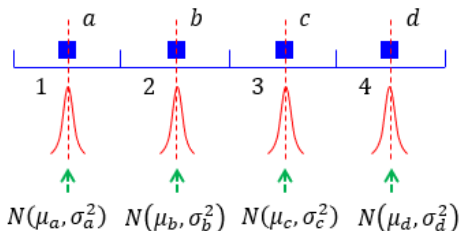

(a)

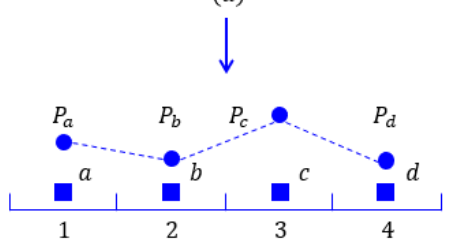

(b)

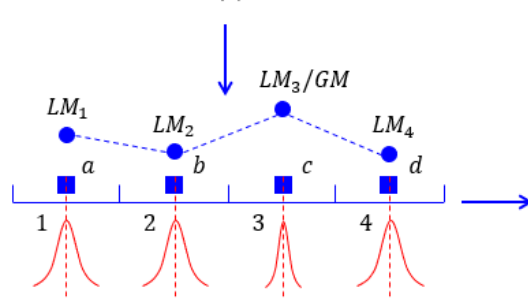

(c)

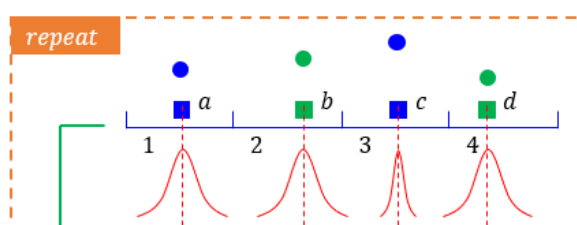

$(f)$

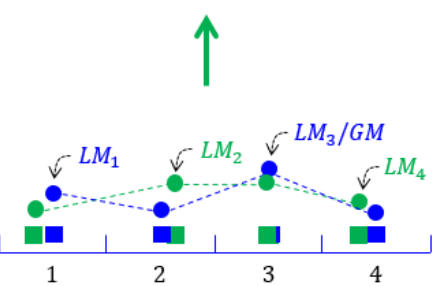

(e)

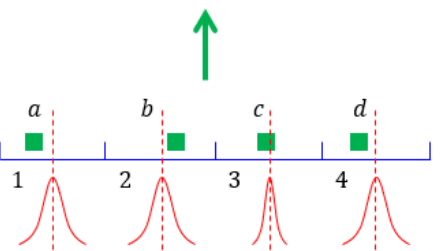

(d)

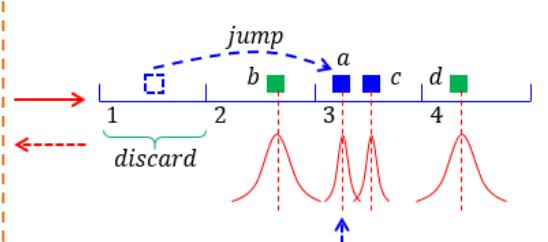

(g) $N\left(\mu_{a}, \sigma_{a}^{2}\right)$

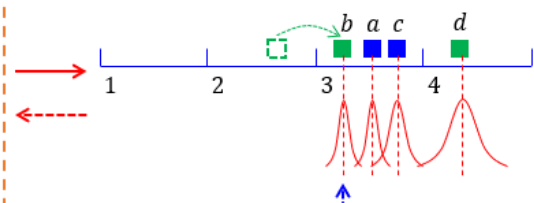

(h) $N\left(\mu_{b}^{\prime}, \sigma_{b}^{2}\right)$

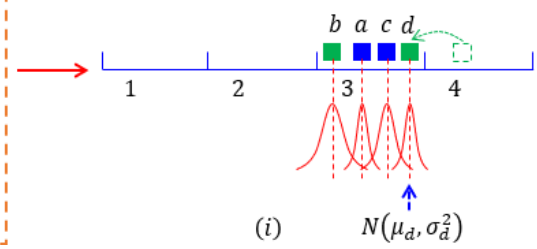

Fig. 3. Particle jump stage (a) Step 1. (b) Step 2. (c) Step 3. (d) Step 4. (e) Step 5. (f) Step 6. (g) Step 7. (h) Step 8. (i) Step 9

traversal is valid while the latter one is invalid. Therefore, it can be concluded that if the particle $b$ can jump over the duty range $d_{a b}$, the tracking time of particle $b$ from the initial position to the global optimal position $c$ can be reduced. Therefore, in this way, the total tracking time of PSO algorithm can be effectively reduced.

4.2 Particle Jump Process Analysis The proposed particle jump method is analyzed in this section, and to simplify the analysis, 4 particles are employed in the PSO algorithm. Furthermore, in order to conduct the particle jump method, the following should be hypothesized: Compared with the $L M$ s, the possibility that the real GM is in the neighborhood area of the current tracked $G M$ is higher. In order to avoid the overlapping of the traversed duty ranges of each particle, the converter duty range is divided into 4 intervals as shown in Fig. 3(a). Then, the particle jump process in Fig. 3 is analyzed in the following.

Step 1: In the algorithm initialization, the 4 particles are placed at the center position of respective intervals. Furthermore, each particle possesses a corresponding Gaussian distribution. In the initialization, the expectations of Gaussian distributions are set as the particle positions and the variances are the same, namely: $\mu_{a}=a, \mu_{b}=b, \mu_{c}=c, \mu_{d}=d$ and $\sigma_{a}^{2}=\sigma_{b}^{2}=\sigma_{c}^{2}=\sigma_{d}^{2}$.

Step 2: At the $1^{\text {st }}$ iteration, the PV output powers of the 4 particles are calculated as shown in Fig. 3(b). Among all the powers, the $L M$ s and $G M$ are determined. For example, at this iteration, the power $P_{c}$ of particle $c$ in the interval 3 is both the $G M$ and the $L M_{3}$. Furthermore, the powers of $P_{a}$, $P_{b}$ and $P_{d}$ of particle $a, b$ and $d$ are the $L M$ s of interval 1,2 and 4 , respectively.

Step 3: In order to search for the potential duties whose power can exceed the current GM, the particles in the interval
1, 2 and 4 for the next iteration should be regenerated in wider ranges. In this paper, because the particle positions are determined by the corresponding Gaussian distributions, the wider range search is conducted by increasing the corresponding distribution variance as shown in Fig. 3(c). For example, because the particle $c$ power $P_{c}$ is the current $G M$, the variance of $N_{c}\left(\mu_{c}, \sigma_{c}^{2}\right)$ is not increased while the other variances are increased. Furthermore, the expectations of the 4 Gaussian distributions are set as the $L M \mathrm{~s}$.

Step 4: With the updated $L M \mathrm{~s}$ and $G M$, the particles for the next iteration are regenerated with the corresponding Gaussian distributions as shown in Fig. 3(d). Because $\sigma_{a}^{2}, \sigma_{b}^{2}$ and $\sigma_{d}^{2}$ are increased, the new particles $a, b$ and $d$ can explore more widely in the corresponding intervals.

Step 5: With the new particles at this iteration, all the particle powers are also calculated. Comparing the current powers with the previous data, the $L M \mathrm{~s}$ and GM should be updated as shown in Fig. 3(e).

Step 6: The variances of the Gaussian distributions whose particle powers are not the $G M$ should be increased, and the expectations should also be updated with the $L M \mathrm{~s}$ as shown in Fig. 3(f). For example, because the particle power $P_{a}, P_{b}$ and $P_{d}$ are not the $G M$, the variances $\sigma_{a}^{2}, \sigma_{b}^{2}$ and $\sigma_{d}^{2}$ will be further increased to obtain more scattered particles for the next iteration. Furthermore, the expectations $\mu_{a}, \mu_{b}$ and $\mu_{d}$ are updated with the $L M_{1}, L M_{2}$ and $L M_{4}$, respectively. After this step, the algorithm will move to the step 4 to repeat the potential particle searching process.

Step 7: With the repeated iterations from step 4 to 6 , the variances of the Gaussian distributions whose particle powers are not the $G M$ will be continuously increased, and there will be an iteration at which the variance is high enough. When the variance is high enough, it can be seen that this interval 


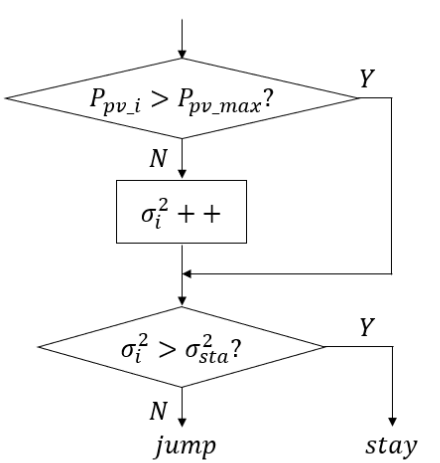

(a)

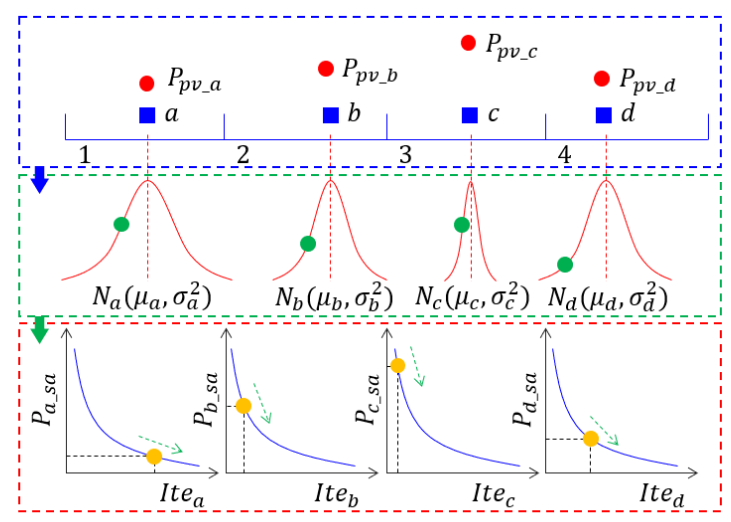

(b)

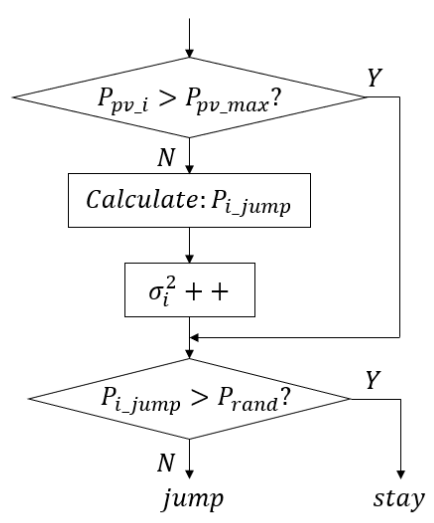

(c)

Fig. 4. (a) Variance decrease flowchart. (b) Proposed SA method. (c) SA flowchart

has been completely traversed and there is no potential particle in the current interval whose power can be higher than the GM. Therefore, this interval should be discarded and the particle of this interval should be moved to other intervals. Because the real $G M$ is the most likely to appear in the interval where the current tracked $G M$ is, the particle whose interval is discarded should jump to the current $G M$ interval.

For example, in Fig. 3(g), the particle $a$ jumps into the interval 3 and the interval 1 is discarded.

Furthermore, the new position of particle $a$ in the interval 3 also should be analyzed. In this paper, the new position of the jumping particle $a$ is generated with the Gaussian distribution of $G M$. In other word, $\mu_{a}$ is generated according to $N_{c}\left(\mu_{c}, \sigma_{c}^{2}\right)$. Furthermore, the Gaussian distribution variance $\sigma_{a}^{2}$ is equal to that of $G M$ Gaussian distribution, namely $\sigma_{a}^{2}=\sigma_{c}^{2}$.

Step 8 and 9: In step 7, the particle $a$ jumps into the interval 3 , and the interval 1 is discarded. Repeat the above steps for enough iterations, and then the rest particles $b$ and $d$ will also jump into the interval where the $G M$ is, namely the interval 3 in this paper.

With the steps 1-9, all the employed particles are moved to the interval where the current tracked GM is, and the particle jump stage will be ended. In the following, the PSO stage will be conducted to track the real GM. Therefore, based on the above analysis, the particle jump stage is used to estimate the rough position of the $G M$ and rapidly reduce the particle distribution range. After the particle jump stage, all the particles will move to the same interval and in order to track the accurate position of $G M$, the PSO stage starts. Compared with the conventional PSO algorithm, the proposed 2-stage PSO method can effectively reduce the tracking time.

\section{Particle Jump Detection Analysis}

As analyzed above, when the particle Gaussian distribution variance is high enough, the corresponding interval should be discarded and the particle should jump into the current $G M$ interval. However, the standard to determine whether the variance is high enough is difficult to be decided. Therefore, in this section, there are two methods are discussed, namely the variance increase method and simulated annealing method.

5.1 Variance Increase Method Because when the variance is high enough the particle should jump, a preset standard $\sigma_{\text {sta }}^{2}$ can be employed to determine whether the current variance is high enough or not. The variance decrease method is shown in Fig.4(a). In details, at each iteration, the current Gaussian distribution variance of each particle is compared with the preset standard, when the variance exceeds the standard, the corresponding particle will jump into the interval where the current global $G M$ is.

For the variance decrease method, the simple complementation is one of the most effective advantages, but the disadvantage is also obvious. The variance standard $\sigma_{\text {sta }}^{2}$ should be carefully determined, because: on one hand, a too high standard will cause long traversal time before the particles jump and then the total tracking time will be prolonged; on the other hand, a low standard indicates that the traversal process is not sufficient and then the potential duties will be missed.

5.2 Simulated Annealing Method Simulated annealing (SA) algorithm is a Monte-Carlo based stochastic optimal point tracking method, and it is based on the temperature decrease of melt iron annealing process. SA algorithm can endow the optimal point tracking process with a probability which gradually decreases to zero. Based on the Metropolis principle, the system will break away from the local optimal positions to achieve the global optimal point tracking.

To conduct the SA algorithm, the following parameters are required: the initial temperature $T_{i n i}$, final temperature $T_{f i}$ and cooling rate $\alpha_{T}$. After the algorithm initialization, under working conditions, the system fitness will be calculated. If the current fitness is higher than the tracked current $G M$, the system state will be updated with the current working point and the system temperature $T_{c u}$ will be reduced for the next algorithm iteration. If the current fitness is lower than the current $G M$, the probability $P$ that the system would accept the current state will be calculated. If the probability is higher than the standard $P_{\text {rand }}$, the current state will be accepted and if it is lower than $P_{\text {rand }}$, the state will be omitted. In this way, the system can break away from some local optimal positions and finally achieve the global optimal tracking.

In this paper, the SA algorithm is employed to decide whether the particle jumps from the current interval into the one where the current $G M$ is. As analyzed above, the particle regeneration for the next iteration is based on the Gaussian distribution of each particle. In the proposed SA method, each particle is arranged a SA module in Fig. 4(b), and in the 


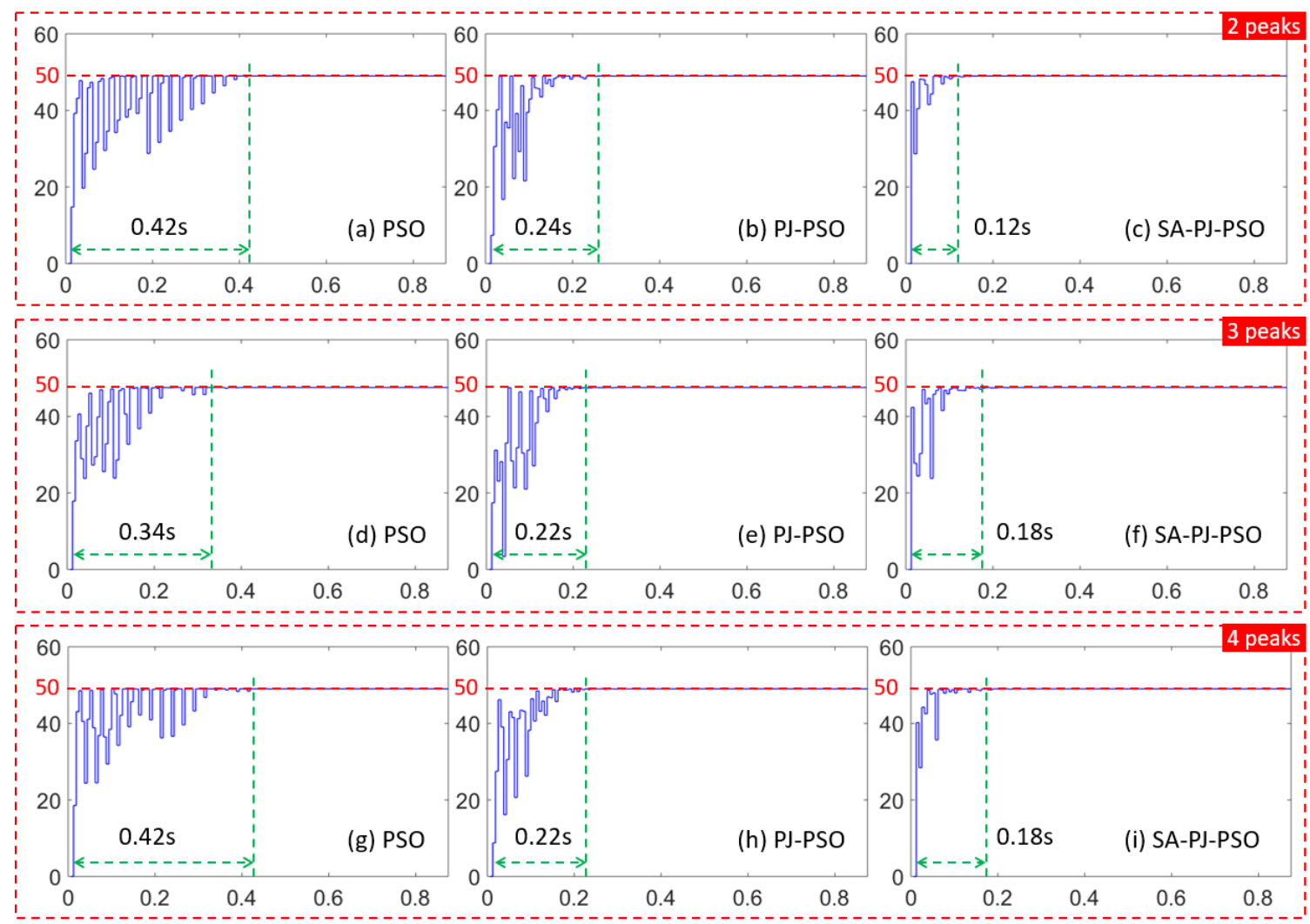

Fig. 5. Simulation results of PSO, PJ-PSO and SA-PJ-PSO algorithms: (a)(b)(c) case 1 of 2 peaks. (d)(e)(f) case 2 of 3 peaks. (g)(h)(i) case 3 of 4 peaks

SA module, the PV output power $P_{p v}$ is employed as the system energy. Therefore, the probability function of each SA module can be obtained as follows.

$$
\begin{aligned}
& P_{i_{-} s a}=e^{\Delta E / T_{i t e_{k}}} \ldots \ldots \\
& \Delta E=P_{p v \_i}-P_{p v \_ \text {max }} \\
& T_{i t e_{k}}=\alpha_{T}^{i t e_{k}} T_{i n i} \ldots \ldots
\end{aligned}
$$

In the equations, $\Delta E$ is the system energy difference; $T_{i t e_{k}}$ is the temperature of the $k^{\text {th }}$ iteration; $i$ stands for the particle $a, b, c$ and $d ; P_{p v_{-} i}$ is the PV output power of particle $i$; $P_{p v \_ \text {max }}$ is the maximum PV power; $P_{i_{-} s a}$ is the probability of that the $i^{\text {th }}$ particle can find the potential GM.

Under working conditions, the regenerated particle will be regarded as a disturbance to the corresponding SA state and the probability $P_{i_{-} s a}$ of each particle can be obtained. In this paper, the particle jump probability $P_{i_{-} \text {jump }}$ is defined as follows.

$$
P_{i_{-} \text {jump }}=1-P_{i_{-}}
$$

The proposed SA method flowchart is shown in Fig. 4(c). Therefore, with algorithm iteration conducting, for the $i^{\text {th }}$ particle, if the PV output power $P_{p v_{-} i}$ is lower than the current $G M$, the particle jump probability $P_{i_{-} \text {jump }}$ will keep exponentially increase. In this way, when $P_{p v_{-} i}<P_{p v \_ \text {max }}$ in the beginning stage, the particle $i$ may not jump, but if it happens in the ending stage, the particle $i$ may jump.

Furthermore, when the PV output power $P_{p v i}<G M$, the particle jump probability $P_{i_{-} \text {jump }}$ are low and high at the beginning and ending stages, respectively. Therefore, with the SA method, the particle jump can be determined with variant probability analysis.

\section{Simulation Verification}

In this paper, in order to verify the proposed algorithm, the 2-peak, 3-peak and 4-peak PV output properties as shown in Fig. 1 are employed in both the simulation and experiment verifications. In this section, the proposed algorithm is verified with Matlab simulation. In all the algorithms, there are 4 particles employed and the initial duties are set as $0.2,0.4$, 0.6 and 0.8 , respectively, and furthermore, the particle duty update upper limit is 0.02. The employed PSO, PJ-PSO and SA-PJ-PSO algorithms parameters are set in the following. In the PSO method, the inertia weight $w$ is set as 0.4, and the acceleration coefficients $c_{1}$ and $c_{2}$ are set as $c_{1}=0.2$ and $c_{2}=2$, respectively; In the PJ-PSO method, the 4 particle position Gaussian distribution expectations are the particle initial duties, respectively, namely: $\mu_{a}=0.2, \mu_{b}=0.4$, $\mu_{c}=0.6$ and $\mu_{d}=0.8$. The initial variances are set as: $\sigma_{a}^{2}=\sigma_{b}^{2}=\sigma_{c}^{2}=\sigma_{d}^{2}=0.1$. Furthermore, in the variance decrease method, the variance standard $\sigma_{\text {sta }}^{2}$ for particle jump is 0.35 and the increment is 0.02 when the particle power is lower than the GM. In the SA-PJ-PSO method, the parameters are set with the same as the PJ-PSO algorithm except the SA algorithm module. The system initial temperature $T_{\text {in }}$ and cooling factor $\alpha_{T}$ are set to be 100 and 0.2 , respectively. The final temperature $T_{f i}$ is set as the temperature when $P_{i_{-} s a}>0.95$. In addition, the frequency of the PSO, PJ-PSO and SA-PJ-PSO is set as $40 \mathrm{~Hz}$.

In order to assess the feasibility of proposed method, the tracking performance can be analyzed mainly in tracking 
time and accuracy aspects. When the PV output power fluctuation is limited within 5\%, the MPPT control is regarded to have been achieved. The time from the moment when the algorithm starts and to the moment when the MPPT is achieved is defined as the algorithm tracking time. Furthermore, the ratio between the power $P_{\text {sta }}$ in the stable state and theory maximum power $P_{\text {max }}$ is taken as the tracking efficiency $\eta_{\text {tra }}$.

The simulation results are shown in Fig. 5 and Table 2, and based on the results, the MPPT control has been achieved in all the cases. When the PV output power possesses 2 peaks with respect to voltage, the MPPT control is acheved within $0.42 \mathrm{~s}, 0.24 \mathrm{~s}$ and $0.12 \mathrm{~s}$ in the methods of PSO, PJPSO and SA-PJ-PSO, respectively. Furthermore, the MPPT efficiency $\eta_{\text {tra }}$ is $99.78 \%, 99.82 \%$ and $99.83 \%$, respectively. Furthermore, the PV output power of PSO method varies intensely in the tracking process, and this is because the particle position in the PSO algorithm is updated gradually iteration by iteration. On the other hand, the power variation of the proposed SA-PJ-PSO algorithm is much less intense due to the fast convergence of particles with the assistance

Table 2. Simulation results of 3 methods

\begin{tabular}{cccccc}
\hline Case & Method & $P_{\text {sta }} / \mathrm{W}$ & $\eta_{\text {tra }}$ & Iteration & Time/s \\
\hline \multirow{3}{*}{1} & PSO & 48.87 & $99.78 \%$ & 17 & 0.42 \\
\cline { 2 - 6 } & PJ-PSO & 48.89 & $99.82 \%$ & 10 & 0.24 \\
\cline { 2 - 6 } & SA-PJ-PSO & 48.90 & $99.83 \%$ & 5 & 0.12 \\
\hline \multirow{3}{*}{2} & PSO & 47.43 & $99.79 \%$ & 14 & 0.34 \\
\cline { 2 - 6 } & PJ-PSO & 47.45 & $99.83 \%$ & 9 & 0.22 \\
\cline { 2 - 6 } & SA-PJ-PSO & 47.46 & $99.85 \%$ & 8 & 0.18 \\
\hline \multirow{3}{*}{3} & PSO & 48.89 & $99.73 \%$ & 17 & 0.42 \\
\cline { 2 - 6 } & PJ-PSO & 48.90 & $99.76 \%$ & 9 & 0.22 \\
\cline { 2 - 6 } & SA-PJ-PSO & 48.93 & $99.82 \%$ & 8 & 0.18 \\
\hline
\end{tabular}

of particle jump and simulated annealing. In the MPPT simulation of 3 peaks, the tracking time of 3 methods is $0.34 \mathrm{~s}$, $0.22 \mathrm{~s}$ and $0.18 \mathrm{~s}$, respectively. Furthermore, the tracking efficiency $\eta_{\text {tra }}$ is $99.79 \%, 99.83 \%$ and $99.85 \%$, respectively. In the MPPT simulation of 4 peaks, the tracking time of 3 methods is $0.42 \mathrm{~s}, 0.22 \mathrm{~s}$ and $0.18 \mathrm{~s}$, respectively. Furthermore, the tracking efficiency $\eta_{\text {tra }}$ is $99.73 \%, 99.76 \%$ and $99.82 \%$, respectively.

Therefore, in case 1, 2 and 3, the proposed SA-PJ-PSO method has achieved both the shortest MPPT time and the least PV output power fluctuation in the tracking process.

\section{Experiment Verification}

The experiment platform is established in Fig. 6. The PSO, PJ-PSO and SA-PJ-PSO algorithms are conducted at $13 \mathrm{~Hz}$ and the upper limit of particle position update step is 0.02 . The PV output property under partial shading conditions in Fig. 1 is achieved with the Keysight E4350B.

The experiment results are shown in Fig. 7 and Table 3. Same to the simulation, the MPPT control has been achieved in all the cases. In case 1 of two peaks, the stable states for the PSO, PJ-PSO and SA-PJ-PSO when the $G M$ positions are

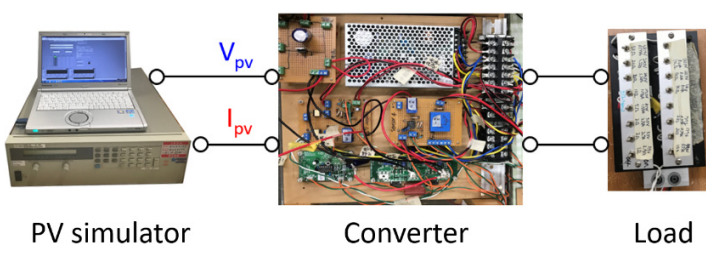

Fig. 6. Experiment platform

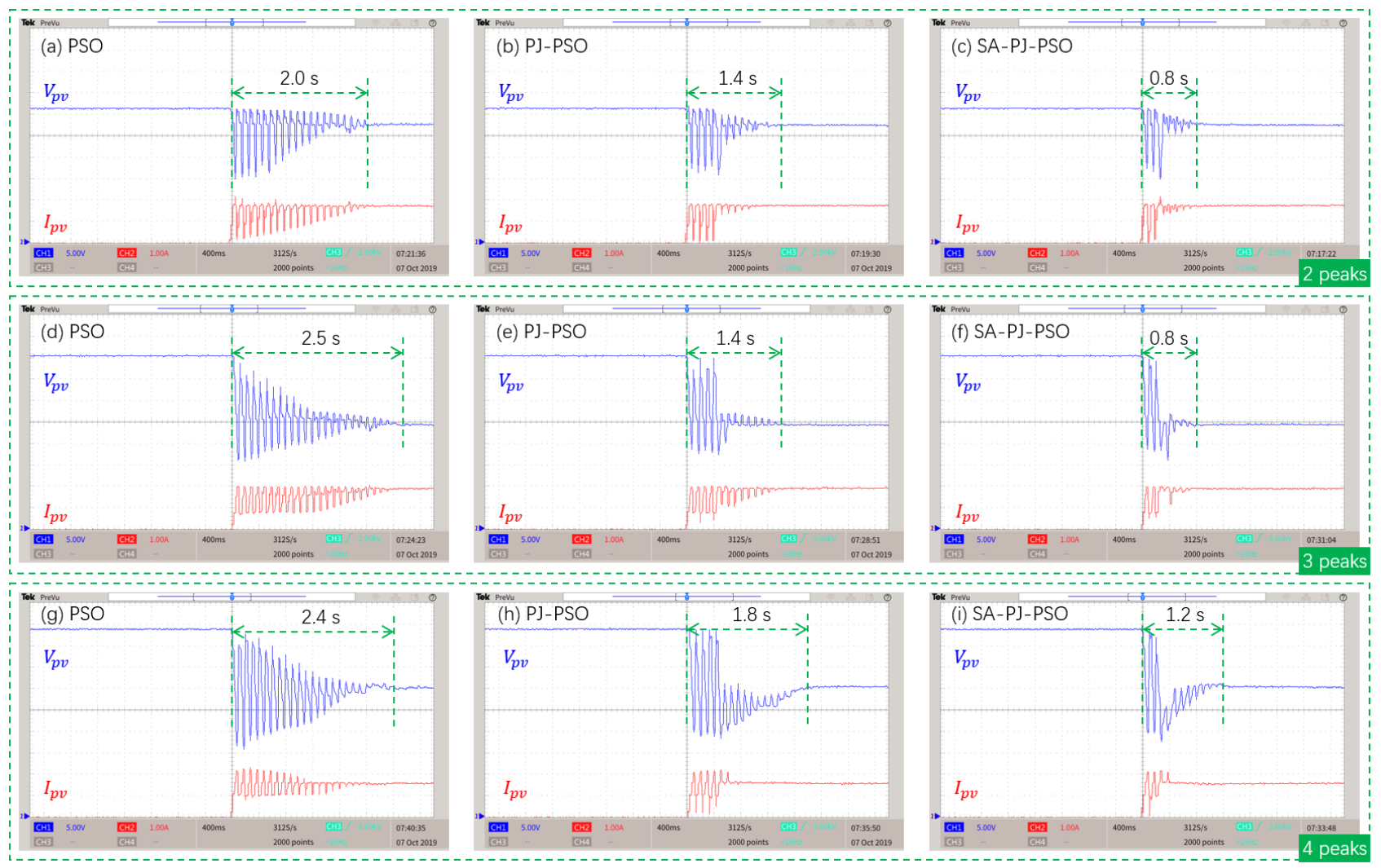

Fig. 7. Experiment results of PSO, PJ-PSO and SA-PJ-PSO algorithms: (a)(b)(c) case 1 of 2 peaks. (d)(e)(f) case 2 of 3 peaks. (g)(h)(i) case 3 of 4 peaks 
Table 3. Experiment results of 3 methods

\begin{tabular}{cccccc}
\hline Case & Method & $P_{\text {sta }} / \mathrm{W}$ & $\eta_{\text {tra }}$ & Iteration & Time/s \\
\hline \multirow{3}{*}{1} & PSO & 47.62 & $97.22 \%$ & 27 & 2.0 \\
\cline { 2 - 6 } & PJ-PSO & 47.81 & $97.61 \%$ & 19 & 1.4 \\
\cline { 2 - 6 } & SA-PJ-PSO & 48.03 & $98.06 \%$ & 11 & 0.8 \\
\hline \multirow{3}{*}{2} & PSO & 46.11 & $97.01 \%$ & 33 & 2.5 \\
\cline { 2 - 6 } & PJ-PSO & 46.54 & $97.92 \%$ & 19 & 1.4 \\
\cline { 2 - 6 } & SA-PJ-PSO & 46.71 & $98.27 \%$ & 11 & 0.8 \\
\hline \multirow{3}{*}{3} & PSO & 47.04 & $95.96 \%$ & 32 & 2.4 \\
\cline { 2 - 6 } & PJ-PSO & 47.69 & $97.29 \%$ & 24 & 1.8 \\
\cline { 2 - 6 } & SA-PJ-PSO & 48.13 & $98.18 \%$ & 16 & 1.2 \\
\hline
\end{tabular}

tracked are achieved within $2.0 \mathrm{~s}, 1.4 \mathrm{~s}$ and $0.8 \mathrm{~s}$, respectively. Furthermore, the MPPT efficiency $\eta_{\text {tra }}$ is $97.22 \%, 97.61 \%$ and $98.06 \%$, respectively. Not only exhibits much better tracking performance than the PSO algorithm, the proposed SA-PJ-PSO algorithm has also possessed advantages over the PJ-PSO algorithm in the MPPT time. Because in the SAPJ-PSO algorithm, the particle jump is decided based on the probability analysis in SA modules, the too lower or higher variance standard $\sigma_{\text {sta }}^{2}$ in PJ-PSO method can be avoided. In the MPPT experiment of 3 peaks, the tracking time of 3 methods is $2.5 \mathrm{~s}, 1.4 \mathrm{~s}$ and $0.8 \mathrm{~s}$, respectively. Furthermore, the tracking efficiency $\eta_{\text {tra }}$ is $97.01 \%, 97.92 \%$ and $98.27 \%$, respectively. In the MPPT experiment of 4 peaks, the tracking time of 3 methods is $2.4 \mathrm{~s}, 1.8 \mathrm{~s}$ and $1.2 \mathrm{~s}$, respectively. Furthermore, the tracking efficiency $\eta_{\text {tra }}$ is $95.96 \%, 97.29 \%$ and $98.18 \%$, respectively.

Therefore, same to the simulation results, in case 1,2 and 3 , the proposed SA-PJ-PSO method has presented the shortest tracking time and the least intensity PV output power fluctuation in the tracking process.

\section{Conclusion}

In this paper, a novel SA-PJ-PSO algorithm has been proposed to track the PV output power $G M$ under partial shading conditions. In order to prevent the particle range which has already been explored to be traversed again, the particle jump method is employed to move the particles over this range to the interval where the current tracked $G M$ is. Furthermore, the SA algorithm is employed to determine whether the particle should jump from the corresponding interval or not. Compared with the conventional methods, the proposed SA-PJPSO algorithm has achieved faster and more stable MPPT performance. Based on the exeriment results, when the PV output property possesses 2,3 and 4 peaks, the tracking time is $0.8 \mathrm{~s}, 0.8 \mathrm{~s}$ and $1.2 \mathrm{~s}$, respectively, verifying the proposed algorithm feasibility.

\section{References}

(1) X. Ju, C. Xu, Y. Hu, X. Han, G. Wei, and X. Du: "A review on the development of photovoltaic/concentrated solar power (PV-CSP) hybrid systems", Solar Energy Materials and Solar Cells, Vol.161, pp.305-327 (2017)

( 2 ) M. Ding, Z. Xu, W. Wang, X. Wang, Y. Song, and D. Chen: "A review on China's large-scale PV integration: Progress, challenges and recommendations", Renewable and Sustainable Energy Reviews, Vol.53, pp.639-652 (2016)

( 3 ) F. Hyder, K. Sudhakar, and R. Mamat: "Solar PV tree design: A review", Renewable and Sustainable Energy Reviews, Vol.82, pp.1079-1096 (2018)

( 4 ) A. Mohapatra, B. Nayak, P. Das, and K.B. Mohanty: "A review on MPPT techniques of PV system under partial shading condition", Renewable and Sustainable Energy Reviews, Vol.80, pp.854-867 (2017)
( 5 ) K.S. Tey, S. Mekhilef, M. Seyedmahmoudian, B. Horan, A.T. Oo, and A. Stojcevski: "Improved differential evolution-based MPPT algorithm using SEPIC for PV systems under partial shading conditions and load variation", IEEE Transactions on Industrial Informatics, Vol.14, No.10, pp.4322-4333 (2018)

( 6 ) M. Seyedmahmoudian, B. Horan, T.K. Soon, R. Rahmani, A.M.T. Oo, S. Mekhilef, and A. Stojcevski: "State of the art artificial intelligence-based MPPT techniques for mitigating partial shading effects on PV systems-A review", Renewable and Sustainable Energy Reviews, Vol.64, pp.435-455 (2016)

( 7 ) M. Seyedmahmoudian, T.K. Soon, B. Horan, A. Ghandhari, S. Mekhilef, and A. Stojcevski: "New ARMO-based MPPT Technique to Minimize Tracking Time and Fluctuation at Output of PV Systems under Rapidly Changing Shading Conditions", IEEE Transactions on Industrial Informatics (2019)

( 8 ) R.J. Prasanth, T. Sudhakar Babu, and N. Rajasekar: "A comprehensive review on solar PV maximum power point tracking techniques", Renewable and Sustainable Energy Reviews, Vol.67, pp.826-847 (2017)

( 9 ) Podder, A. Kumer, N. Kumar Roy, and H. Roy Pota: "MPPT methods for solar PV systems: a critical review based on tracking nature", IET Renewable Power Generation, Vol.13, pp.1615-1632 (2019)

(10) Jordehi, A. Rezaee: "Maximum power point tracking in photovoltaic (PV) systems: A review of different approaches", Renewable and Sustainable Energy Reviews, Vol.65, pp.1127-1138 (2016)

(11) Koad, Ramdan BA, Ahmed Faheem Zobaa, and Adel El-Shahat: "A novel MPPT algorithm based on particle swarm optimization for photovoltaic systems", IEEE Transactions on Sustainable Energy, Vol.8, pp.468-476 (2016)

(12) H. Li, D. Yang, W. Su, J. Lu, and X. Yu: "An overall distribution particle swarm optimization MPPT algorithm for photovoltaic system under partial shading", IEEE Transactions on Industrial Electronics, Vol.66, No.1, pp.265-275 (2018)

(13) K. Sundareswaran and S. Palani: "Application of a combined particle swarm optimization and perturb and observe method for MPPT in PV systems under partial shading conditions", Renewable Energy, Vol.75, pp.308-317 (2015)

(14) Diab, Ahmed A. Zaki, and Hegazy Rezk: "Global MPPT based on flower pollination and differential evolution algorithms to mitigate partial shading in building integrated PV system", Solar Energy, Vol.157, pp.171-186 (2017)

(15) N. Priyadarshi, S. Padmanaban, L. Mihet-Popa, F. Blaabjerg, and F. Azam: "Maximum power point tracking for brushless DC motor-driven photovoltaic pumping systems using a hybrid ANFIS-FLOWER pollination optimization algorithm”, Energies, Vol.11, No.5, pp.1067-1083 (2018)

(16) K.S. Ey, S. Mekhilef, M. Seyedmahmoudian, B. Horan, A.T. Oo, and A. Stojcevski: "Improved differential evolution-based MPPT algorithm using SEPIC for PV systems under partial shading conditions and load variation", IEEE Transactions on Industrial Informatics, Vol.14, No.10, pp.4322-4333 (2018)

(17) Diab, Ahmed A. Zaki, and Hegazy Rezk: "Global MPPT based on flower pollination and differential evolution algorithms to mitigate partial shading in building integrated PV system", Solar Energy, Vol.157, pp.171-186 (2017)

(18) A.S. Oshaba, E.S. Ali, and SM Abd Elazim: "PI controller design using ABC algorithm for MPPT of PV system supplying DC motor pump load", Neural Computing and Applications, Vol.28, No.2, pp.353-364 (2017)

(19) K. Sundareswaran, V. Vethanayagam, and P. Sankaran: "Development of a hybrid genetic algorithm/perturb and observe algorithm for maximum power point tracking in photovoltaic systems under non-uniform insolation”, IET Renewable Power Generation, Vol.9, No.7, pp.757-765 (2015)

(20) D.F. Teshome, C.H. Lee, Y.W. Lin, and K.L. Lian: "A modified firefly algorithm for photovoltaic maximum power point tracking control under partial shading", IEEE Journal of Emerging and Selected Topics in Power Electronics, Vol.5, No.2, pp.661-671 (2016)

(21) J.Y. Shi, F. Xue, Z.J. Qin, L.T. Ling, T. Yang, Y. Wang, and J. Wu: "Tracking the global maximum power point of a photovoltaic system under partial shading conditions using a modified firefly algorithm", Journal of Renewable and Sustainable Energy, Vol.8, No.3, pp.033501 (2016)

(22) S. Mohanty, B. Subudhi, and P.K. Ray: "A grey wolf-assisted perturb \& observe MPPT algorithm for a PV system”, IEEE Transactions on Energy Conversion, Vol.32, No.1, pp.340-347 (2016)

(23) B. Yang, T. Yu, H. Shu, D. Zhu, N. An, Y. Sang, and L. Jiang: "Energy reshaping based passive fractional-order PID control design and implementation of a grid-connected PV inverter for MPPT using grouped grey wolf optimizer”, Solar Energy, Vol.170, pp.31-46 (2018)

(24) P.S. Gavhane, S. Krishnamurthy, R. Dixit, J.P. Ram, and N. Rajasekar: "ELPSO based MPPT for solar PV under partial shaded condition", Energy Procedia, Vol.117, pp.1047-1053 (2017)

(25) J.P. Ram and N. Rajasekar: "A new global maximum power point tracking technique for solar photovoltaic (PV) system under partial shading conditions (PSC)", Energy, Vol.118, pp.512-525 (2018) 
(26) N. Kumar, I. Hussain, B. Singh, and B.K. Panigrahi: "MPPT in dynamic condition of partially shaded PV system by using WODE technique", IEEE Transactions on Sustainable Energy, Vol.8, No.3, pp.1204-1214 (2017)

(27) R. Ramaprabha, V. Gothandaraman, K. Kanimozhi, R. Divya, and B.L. Mathur: "Maximum power point tracking using GA-optimized artificial neural network for solar PV system", 2011 1st International Conference on Electrical Energy Systems. IEEE (2011)

(28) K. Sundareswaran, P. Sankar, and P. Sankaran: "MPPT of PV systems under partial shaded conditions through a colony of flashing fireflies", IEEE Transactions on Energy Conversion, Vol.29, pp.463-472 (2014)

(29) S. Mohanty, B. Subudhi, and P.K. Ray: "A grey wolf-assisted perturb \& observe MPPT algorithm for a PV system", IEEE Transactions on Energy Conversion, Vol.32, No.1, pp.340-347 (2016)

(30) A. Laudani, F.R. Fulginei, and A. Salvini: "Identification of the onediode model for photovoltaic modules from datasheet values", Solar Energy, Vol.108, pp.432-446 (2014)

Bingcheng Ji (Non-member) received the B.S. and M.S. degrees

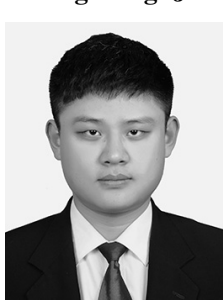
in electrical engineering from Dalian University of Technology, Dalian, China, in 2011 and 2015, respectively. He is currently working toward the Ph.D. degree in the Graduate School of Frontier Sciences, The University of Tokyo, Chiba, Japan. His research interests include wireless power transfer, PV MPPT control and power electronics.

Katsuhiro Hata

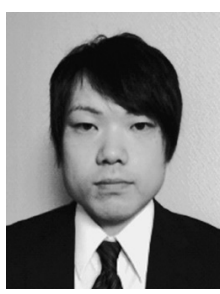

(Member) received the M.S. and Ph.D. degrees from the University of Tokyo in 2015 and 2018, respectively. He is currently a Research Associate with the Department of Informatics and Electronics, Institute of Industrial Science, the University of Tokyo. His research interests include power electronics, wireless power transfer, and e-mobility for transportation. $\mathrm{He}$ is a member of the Institute of Electrical and Electronics Engineers, the Institute of Electronics, Information and Communication Engineers, and the Society of Automotive Engineers of Japan.

Takehiro Imura (Member) received the B.S. degrees in electrical

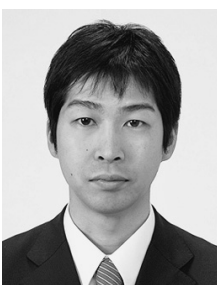
and electronics engineering from Sophia University, Tokyo, Japan, in 2005, and the M.E. degree in electronic engineering and the Ph.D. degree in electrical engineering from the University of Tokyo, in 2007 and 2010, respectively. He joined the Department of Advanced Energy, Graduate School of Frontier Sciences, the University of Tokyo, as a Research Associate in 2010, he moved to the Department of Electrical Engineering and Information Systems, Graduate School of Engineering, the University of Tokyo as a Project Lecturer. In 2019, he moved to the Department of Electrical Engineering, Faculty of Science and Technology, Tokyo University of Science, as an Associate Professor. He is currently investigating wireless power transfer for EV using magnetic resonance coupling.
Yoichi Hori (Fellow) received the B.S., M.S., and Ph.D. degrees in

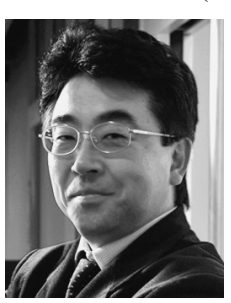
electrical engineering from the University of Tokyo, Tokyo, Japan, in 1978, 1980, and 1983, respectively. In 2000, he became a Professor at the University of Tokyo, and where in 2008, he moved to the Department of Advanced Energy, Graduate School of Frontier Sciences. His research fields are control theory and its industrial applications in motion control, mechatronics, robotics, and electric vehicles. Dr. Hori received the Best Transactions Paper Award from the IEEE Transactions on Industrial Electronics in 1993 and 2001, and the 2011 Achievement Award from the Institute of Electrical Engineers of Japan (IEE-Japan). He was the President of the Industry Applications Society the IEEJ, the President of Capacitors Forum, and the Chairman of the Motor Technology Symposium of the Japan Manahement Association, and the Director on Technological Develipment of SAEJapan.

Sayuri Honda (Non-member) received the B.S. and M.S. degrees in electrical engineering from The University of Tokyo in 2013 and 2015 respectively. She is currently researching power control system of the spacecrafts in JAXA R\&D directorate. Her main research theme is wireless power transmission system for spacecrafts.

Shuhei Shimada (Non-member) received the B.S. and M.S. degrees

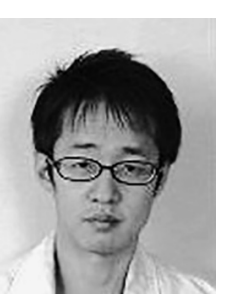
in electrical engineering from Tokyo University of Science in 2007 and 2009 respectively. He is currently developing satellite power systems and small batteries in JAXA R\&D directorate. He researches wireless power supply system for spacecraft.

Osama Kawasaki (Non-member) received the B.S. and M.S. degrees

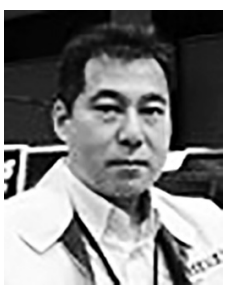
in nuclear engineering from the Osaka University, Osaka, Japan, in 1989 and 1991 respectively. After he entered JAXA, he engaged in the development of the electrical power system of Japanese unmanned transfer vehicle to the International Space Station, HTV, and the asteroid explorer, Hayabusa2.He currently researches the space application of wireless power transfer technique in $\mathrm{R} \& \mathrm{D}$ directorate. 